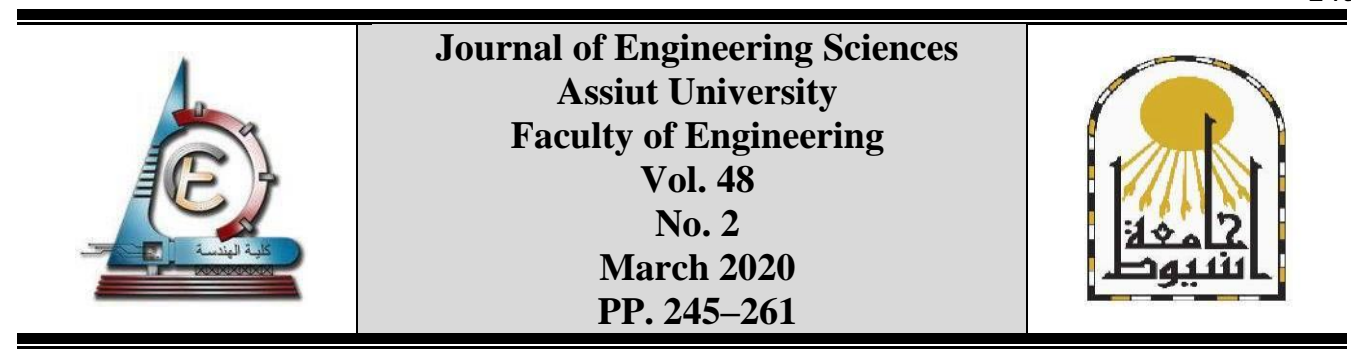

\title{
ARCHITECTURAL COMPARATIVE ANALYSES BETWEEN ROBOTIC SURGERY WARDS IN DIGITAL HOSPITALS AND TRADITIONAL SURGERY WARDS IN CONVENTIONAL HOSPITALS
}

\author{
Randa M. A. Mahmoud ", Amr M. A. Youssef, Nady M. Abdel Karim \\ Department of Architectural Engineering, Assiut University, Assiut 71518, Egypt.
}

Received 22 July 2019; Accepted 16 September 2020

\begin{abstract}
Recently, the integration between healthcare services and new technologies has been enhanced to be very necessary and effective inside digital Hospitals. Digital hospitals include a huge number of healthcare advanced technologies that have special digital and architectural requirement; these requirements cannot be provided in traditional hospitals. Many previous studies and guidelines addressed few numbers of digital hospital's rooms and their architectural requirements. Hence, in this study, healthcare advanced technologies has been determined for outlining the architectural consideration of digital hospital's rooms. Accordingly, Robotic Surgery Ward (RSW) has been compared with Traditional Surgery Ward (TSW) for: a) demonstrating the effect of an advanced technology (Robotic technology) on a digital hospital ward and b) helping designers to find out the main architectural and economic principles of designing RSWs besides TSWs. The main findings in this study are: a) outlining the main architectural characteristics of digital hospitals in general and digital hospital's rooms in specific, b) articulating the main architectural and economic aspects for RSW and robotic surgery rooms, which is different from TSW. As an application of the comparative analyses, possible design alternatives of RSW and TSW has been also proposed and compared.
\end{abstract}

Keyword: Digital Hospital design, Healthcare advanced technologies, Robotic Surgery Ward design, Architectural and economic considerations.

\section{Introduction}

The use of information and communication systems in healthcare services for the diagnosis, treatment, monitoring of diseases and the provision of health counseling is described as "e-Health"; a digital hospital is defined as a sub-component of e-Health [1]. Hence, the digital hospital is a hospital that improves healthcare quality and increases patient satisfaction by implementing Information Technology (IT) infrastructure; this will integrate all kinds of communication tools and medical equipment with each other, with other information systems, healthcare staff and patients. Digital hospital can expand the healthcare services by providing the digital connectivity and collaboration with healthcare

* Correponding author.

E-mal addres: randa.m@aue.edu.eg 
staff in other remote hospitals or with patients inside their houses directly [2]. So, the outcomes of digital hospitals demonstrate that their efficiency increases by $35 \%$ because of the wide range of patients they serve [1]. Digital hospitals include a huge number of healthcare advanced technologies that have special digital and architectural requirement; these requirements cannot be provided in traditional hospitals. Due to the wide variety of healthcare advanced technologies, this paper aims at comparing the architectural and economic aspects of Robotic Surgery ward (RSW) and Traditional Surgery ward (TSW), this also includes the effect of healthcare advanced technologies on the design of digital hospital in general and their internal rooms in specific. Moreover, a set of possible design alternatives for both RSW and TSW have been outlined as a detailed application.

However, digital hospital can provide faster and safer service for patients, while reducing costs. Within previous studies, the various benefits for digital hospital have been addressed. For example, Reffat [2] concluded the main benefits of digital hospitals as: a) handling twice as many patients with a higher level of care without increasing hospital's staff or size, b) improving patient outcomes by reducing length of hospital stay and c) achieving efficiencies of diagnosis and treatment for patients within the shortest time. Also, Kilic [1] illustrated the digital hospital's benefits have been represented as: a) Closed Loop Drug Delivery system for the right medicine to the right patient, b) Real-time location services track medical assets, equipment, patients, and staff to improve patient care, c) digital integration, automation of medical information systems and eHealth Networks. Korea digital Hospital Export Agency [3] concluded other benefits for digital hospital as: a) health data that can be forwarded via sensors, cameras and early warning systems without requiring follow-up by humans and b) efficiencies of medical technologies as digitalized medical equipment, diagnosis and treatment, so, digital hospital is safer and healthier. Courbis [4] outlined that digital hospital stuff has less workload, documentation and administration effort, so the result is increasing staff satisfaction and economic efficient.

On the other hand, numerous studies focused on the challenges which face digital hospitals within their design and establishing process, or during operation and renewal stages. Courbis [4] concluded the challenges of digital hospital such as: the high cost of the digital medical equipment, IT infrastructure and the additional construction cost ranges from $5 \%$ to $10 \%$ for the building. Reffat [2] studied the rapid development of medical equipment considerations and its need for a flexible design of space for ease of use. As well as, Kilic [1] illustrated the difficulty of accreditation and assessment of the digital hospital to receive a "Digital hospital" certificate. Whereas, to be promoted as a digital hospital, a certificate by the accrediting agency Healthcare Information and Management Systems Society (HIMSS) must be assessed and awarded. HIMSS uses the universally accepted accreditation and standard model EMRAM (Electronic Medical Record Adoption Model) to assess the digital processes and determine the stages of applicant hospitals.

Healthcare Advanced Technologies have been determined in various studies, for example, Sprow [5] determined the common healthcare advanced technologies are: robotic surgery, telemedicine and Electronic Medical Record and others. Some studies have acknowledged the effect of the healthcare advanced technologies on some rooms in digital hospital. For example, Martin [6] illustrated that, telemedicine technology requires new room at quit location, without windows, light colors for wall print and Information and Communications Technologies supplies for telemedicine Carts. Also, Kenyon [7] found that Electronic Medical Record technology provides wide area of archive stores and administration can reach up to $1000 \mathrm{~m}^{2}$ as at Henry Ford hospital in Michigan, USA ${ }^{[*]}$. 
Randa M. A. Mahmoud et al., Architectural comparative analyses between robotic surgery wards ......

[*] Henry Ford Hospital (HFH) is an 877-bed tertiary care hospital, education and research complex in USA. It was one of the first to begin using electronic patient records to ensure the highest quality and safest patient care, as well to convert to digital hospital [7].

Numerous studies focused on the influence of robotic surgery technology in the design of robotic surgery rooms. The main considerations of the digital robotic operating rooms (ORs) and relevant equipment have been concluded by Matthew et al. [8]. Also, Kpodonu [9, 10], Rostenberg et al. [11, 12] and Emergency Response Centre International Institute [13] studied the architectural considerations of cardiothoracic hybrid ORs, and Endovascular hybrid ORs. For Neurosurgery hybrid ORs, Gow et al. [14] determined its size, robots, imaging system and relevant equipment of, while Michael et al. [15] focused on both Neurosurgery hybrid ORs and Orthopedics hybrid OR architectural considerations. As well as, few international design guidelines also addressed more specific design considerations for digital hospital spaces such as Health Authority Abu Dhabi guidelines [16] and the Facility Guidelines Institute [17]. The detailed architectural comparison between RSW and TSW in design cases (the whole ward, room details, requirements, main zones in architectural plans and others) were not found in the literature.

Accordingly, this paper addressed the general design principles of digital hospital and their rooms in section 2. In section 3, the paper presented comparative analyses between RSW and TSW, either on the scope of wards or rooms. In section 4, proposed possible design alternatives of RSWs and TSWs have been presented as an application.

\section{The main design principles of the digital hospital and the influence of healthcare advance technology}

In a digital hospital, various healthcare advanced technologies provide a fully integrated set of applications and medical services. Thus, IT infrastructure technologies and devices should be considered from the beginning of the architectural design process of a Digital hospital. So, the four main design principles for digital hospital essentially depended on IT infrastructure are as follows $[4,5]$ :

a) Hospital space rationalization by using IT infrastructure technologies to separate between patient and administration area using the "paperless system"; it leads to the disappearance of all storage or archiving areas.

b) Establishment of the IT infrastructure and medical information system such as Picture Archive and Communication System and Electronic Medical Record.

c) Automating the infrastructure of Support-Services such as automation of the pharmacy unit.

d) A smart building that improve energy consumption

Also, the integration of the healthcare advanced technologies inside the digital hospital gave it special architectural characteristics; the most common architectural characteristics and considerations of digital hospital based on the literature are as follows $[1,2,3,4,5,7,18,19]$ :

a) In the scope of the whole digital hospital and their departments:

- Most common model types for digital hospital are Nucleus model and separated blocks model, which are linking by IT network.

- New departments in digital hospital have been added such as: Robotic surgery ward, Pathology lab, Angiography and Cardiac Catheterization lab, Nuclear 
medicine, Satellite pharmacy, Picture archiving and communication system unit, IT center, Robotic surgery simulation center.

- Modular units for flexibility have been used to cater for digital hospital changes inside wards.

- Multi solutions for Digital hospital wards have been improved as result of digital communication.

- The department's area has been increased according to biotechnology and robotics requirements.

b) In the scope of some rooms of digital hospital:

- Expansion area has been added to rooms with digital equipment..

- Digital equipment's area ratio has been increased from $4 \%$ to $54 \%$ and nondigital equipment's area ratio has been reduced from $96 \%$ to $46 \%$ in last century.

- Medical IT infrastructure should be included, such as Electronic Medical Record, Picture Archive and Communication System, Physiological monitoring systems, Closed-loop medication management, ICT control access, Interactive digital way finding signage, Integrated nurse call, Real-Time Location System, Telemedicine service, Tele surgery service and Tele intensive care service.

- New digital spaces have been added, such as robotic surgery rooms, patient smart room, digitally integrated operating room, Automatic pharmacy, Digital Cath Lab, Smart exam room and Check-in kiosk.

- Medical equipment should been included, such as Digital imaging equipment, laboratory automation, sterile processing equipment and Radio therapy digital equipment.

Therefore, it is necessary to study the common of healthcare advanced technologies, either are digital techniques or digital medical equipment to extract the new rooms at digital hospital or current ones incorporate digital techniques within them. Hence, some rooms at digital hospital with the determining of room type, rooms' equipment, architectural considerations and the spatial relations have been illustrated in Table 1. In addition to that, the main architectural considerations of rooms and area requirement can be determined from the international guidelines of healthcare building as Health Authority Abu Dhabi guidelines [16] and the Facility Guidelines Institute [17]. However, robotic surgery ward has been selected as one of the best wards that demonstrate integrating healthcare advanced technologies, so the design of RSWs and its difference with TSWs will be addressed below. 
Randa M. A. Mahmoud et al., Architectural comparative analyses between robotic surgery wards .......

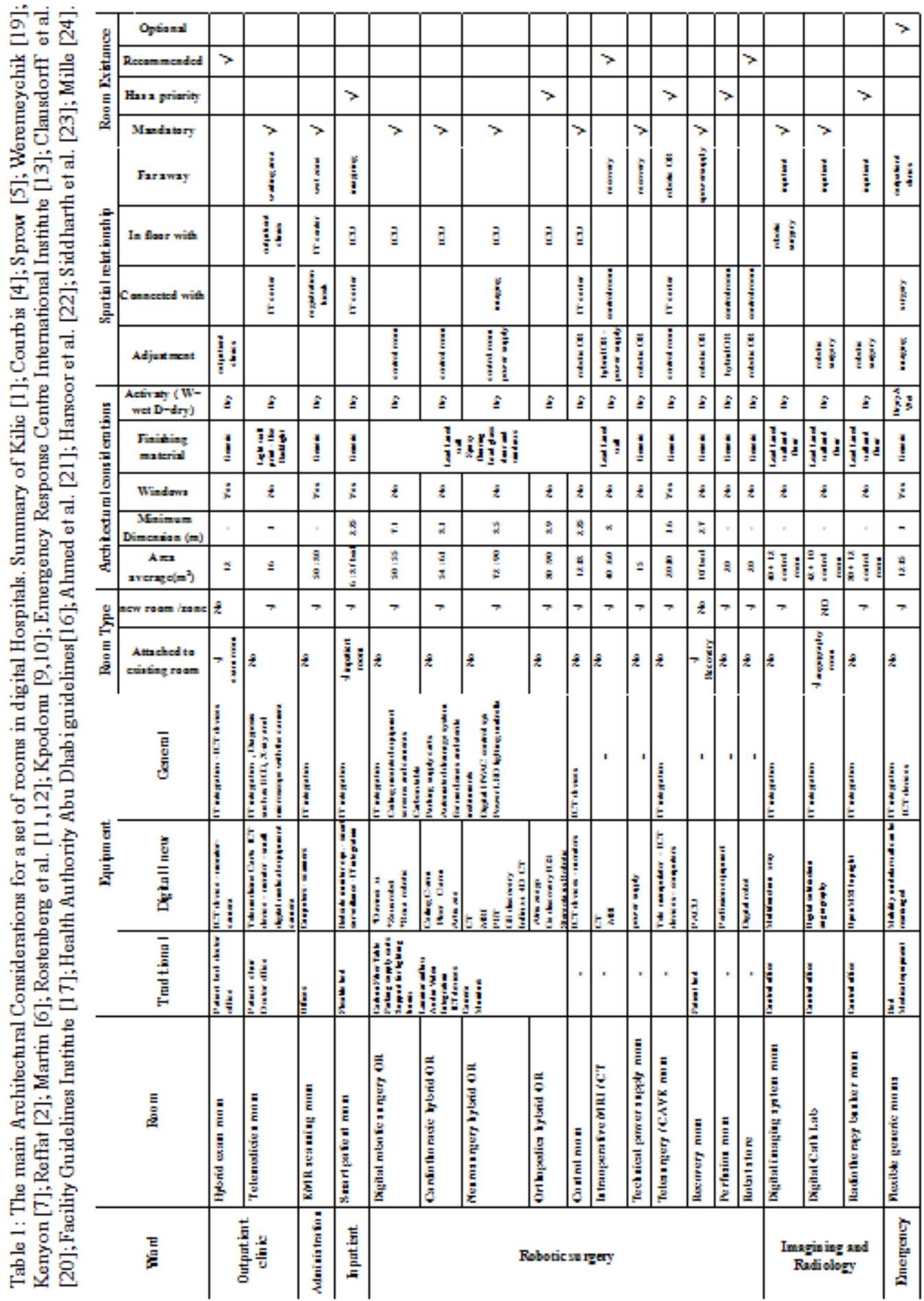


Table 1. Cont.

\begin{tabular}{|c|c|c|c|c|c|c|c|c|c|c|c|c|c|c|c|c|c|c|c|c|c|c|}
\hline \multirow{4}{*}{ 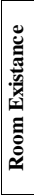 } & Optional & & & & & & & & & & & & $>$ & & & & & & & & & $>$ \\
\hline & Recommended & & & & & & & $>$ & & & & & & & & & $>$ & $>$ & & $>$ & $>$ & \\
\hline & Has a priority & & $>$ & & & & & $>$ & & & $>$ & & & $>$ & & & & & $>$ & & & \\
\hline & Mandatory & $>$ & & $>$ & $>$ & $>$ & $>$ & & $>$ & $>$ & & $>$ & & & $>$ & & & & & & & \\
\hline \multirow{4}{*}{ 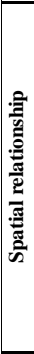 } & Far away & 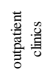 & & 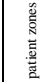 & 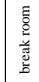 & 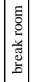 & & 递 & 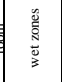 & 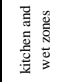 & & & & 喜 & & & & \multirow{4}{*}{ 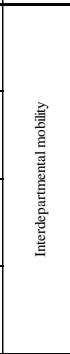 } & & & & \\
\hline & In floor with & & & 总 & & & & & & & & 望 & 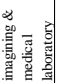 & & & & & & & 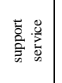 & & \\
\hline & \begin{tabular}{|l|} 
Connected with \\
\end{tabular} & 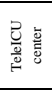 & 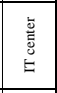 & 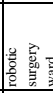 & & & 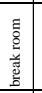 & 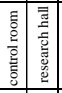 & & 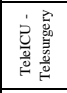 & & $\begin{array}{l}\text { 产 } \\
\stackrel{5}{E} \\
\qquad\end{array}$ & 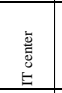 & & 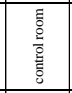 & $\begin{array}{l}\text { 氮 } \\
\text { E } \\
\Xi\end{array}$ & 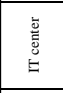 & & & 产产 & 喜 & 害喜 \\
\hline & Adjustment & 璦高 & $\underline{z}$ & 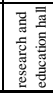 & 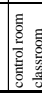 & & 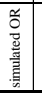 & 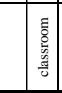 & $\begin{array}{l}\text { 产 } \\
\stackrel{5}{E} \\
\end{array}$ & $\begin{array}{l}\frac{2}{2} \\
\frac{2}{6} \\
\frac{2}{2} \\
\frac{8}{2} \\
\end{array}$ & 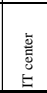 & 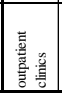 & 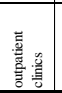 & 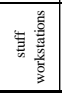 & 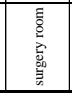 & $\begin{array}{l}\text { 间 } \\
\text { 前 }\end{array}$ & Z & & 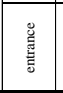 & & 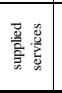 & \\
\hline \multirow{5}{*}{ 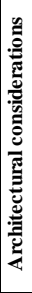 } & $\begin{array}{c}\text { Activaty ( } W= \\
\text { wet } \mathrm{D}=\mathrm{dry} \text { ) }\end{array}$ & I & I & 死 & 8 & 8 & 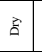 & 20 & ह & s & s & ' & ' & $\cdot$ & ' & ' & ' & ' & ' & ' & ' & ' \\
\hline & $\begin{array}{c}\text { Finishing } \\
\text { material }\end{array}$ & 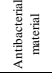 & 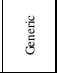 & 竞离 & 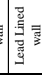 & 焉 & 童 & 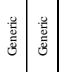 & 害 & 善 & 䜌 & ' & ' & ' & ' & ' & ' & ' & ' & ' & ' & ' \\
\hline & Windows & $\stackrel{\gtrless}{z}$ & $\stackrel{\overbrace 0}{\varkappa}$ & . & そ & 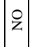 & $\stackrel{s}{\sim}$ & 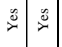 & $\stackrel{\circ}{z}$ & $\stackrel{2}{z}$ & $\AA$ & $\cdot$ & ' & ' & . & . & . & . & ' & . & . & , \\
\hline & \begin{tabular}{|c|} 
Minimum \\
Dimension $(\mathbf{m})$
\end{tabular} & m & . & & 足 & + & - & $m$ & & . & $\cdot$ & . & & . & 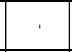 & . & . & . & . & . & . & . \\
\hline & \begin{tabular}{|c|} 
Area average \\
$\left(\mathbf{m}^{2}\right)$
\end{tabular} & $\stackrel{\infty}{\underline{I}}$ & $\frac{8}{8}$ & 总 & 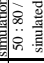 & 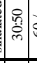 & 8 & 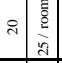 & 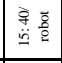 & \&్ల & 8 & . & . & 淟 & . & . & . & $\frac{\mathscr{0}}{\frac{5}{5}}$ & $a$ & . & $\begin{array}{l}11 \\
\text { II } \\
\text { 善 } \\
\end{array}$ & $a$ \\
\hline \multirow{2}{*}{ 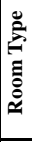 } & new room /zone & $\AA$ & $>$ & $>$ & $>$ & $>$ & $>$ & $>>$ & $>$ & $>$ & $>$ & 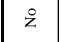 & 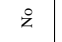 & そ & $\stackrel{2}{z}$ & 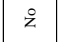 & $\stackrel{\circ}{z}$ & 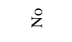 & 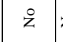 & $\AA$ & そ̊ & $\stackrel{\circ}{z}$ \\
\hline & \begin{tabular}{|c|} 
Attached to \\
existing room
\end{tabular} & 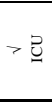 & 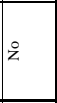 & そ̊ & z & 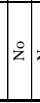 & そ̊ & $\AA \AA$ & $\stackrel{2}{z}$ & 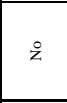 & 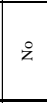 & 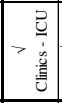 & 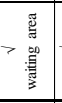 & 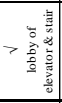 & \begin{tabular}{|r|}
\multicolumn{2}{|c|}{} \\
$>$ 耪 \\
\end{tabular} & 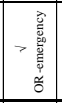 & $>Z$ & 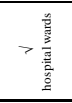 & $>$ 惫 & $>$ 曾 & 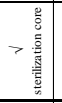 & 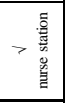 \\
\hline \multirow{3}{*}{ | } & 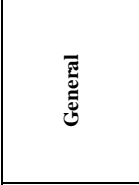 & 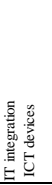 & 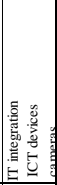 & 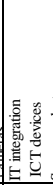 & & & & & 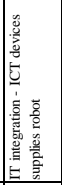 & 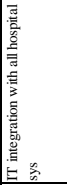 & & 总 & 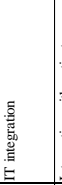 & 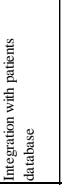 & 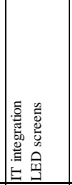 & 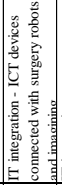 & 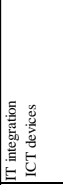 & 总 & 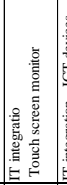 & 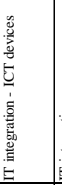 & 总 & 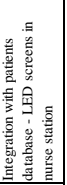 \\
\hline & 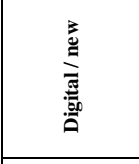 & 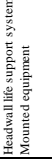 & 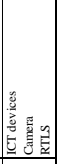 & 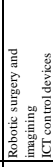 & 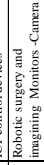 & 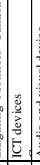 & 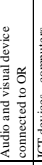 & 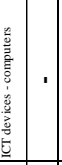 & 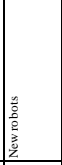 & 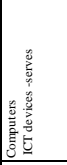 & 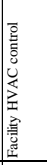 & 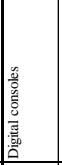 & 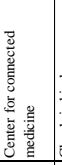 & 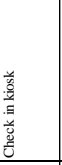 & $\begin{array}{l}0 \\
\substack{0 \\
2 \\
2} \\
\end{array}$ & 言 & $\underline{3}$ & 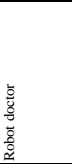 & 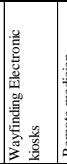 & 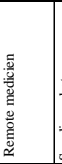 & 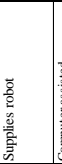 & 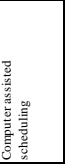 \\
\hline & 矛 & 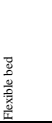 & 愛 & 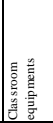 & 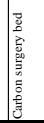 & & 部 & 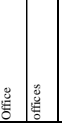 & ' & 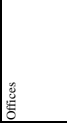 & ' & ' & ' & ' & ' & ' & ' & ' & ' & ' & ' & ' \\
\hline & 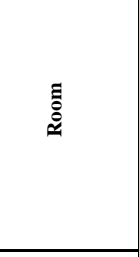 & 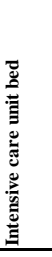 & 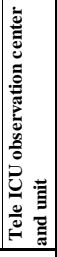 & 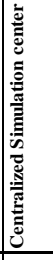 & 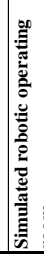 & : & 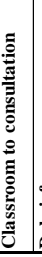 & 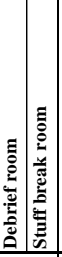 & 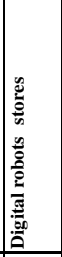 & 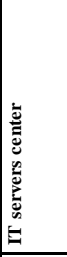 & 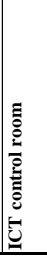 & \multicolumn{11}{|c|}{ Digital Technical don't need to room } \\
\hline & Ward & $\begin{array}{r}\text { Inter } \\
\text { care } \\
\text { IC }\end{array}$ & $\begin{array}{l}\text { nsive } \\
\text { unit } \\
\text { U }\end{array}$ & Medi & $\begin{array}{r}\text { ical } \mathbf{F} \\
\mathbf{R e}\end{array}$ & $\begin{array}{l}\text { Educ } \\
\text { esear }\end{array}$ & $\begin{array}{l}\text { catio } \\
\text { rch }\end{array}$ & on and & 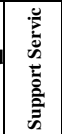 & \multicolumn{2}{|c|}{ IT center } & \multicolumn{11}{|c|}{ Digital Technical for IT infrastructure } \\
\hline
\end{tabular}

* Correponding author.

E-mal addres: randa.m@aue.edu.eg 
Randa M. A. Mahmoud et al., Architectural comparative analyses between robotic surgery wards ......

\section{Architectural comparative analyses between robotic and traditional surgery}

Through the robotic surgery, the surgeon uses one of two methods to control the robots, either via a direct tele-manipulator or through computer control. However, the comparative analyses outcomes between RSW and TSW are: a) demonstrating of the effect of an advanced technology (Robotic technology) on a digital hospital ward and b) helping designers to find out the main architectural and economic principles of designing RSWs besides TSWs. The impact of using robotics in surgery affects the design of both ORs and spaces where robotic devices are stored when not in use. The size of robots requires additional floor space be allocated to both the ward and OR, and their utility requirements introduce a set of cables and power supplies and information and communications technologies devices $[11,12]$. To design the OR of robotic surgery, robots and imaging equipment location should be identified, then other equipment locations and requirements, e.g. ceiling lights, surgical and video integration surgery workflow can be determined. Robotic surgeries require specific types of ORs: a) digital integrated OR and b) Hybrid OR. A digital integrated OR is defined as an operating room that is equipped with the necessary equipment (booms, lights, routers, touch panels, device control, capture systems, etc.) to facilitate the flow of communication, data, video and the overall interaction of the healthcare providers with the patient (Akridge [25]). A Hybrid OR is a surgical OR that is equipped with advanced medical imaging equipment such as fixed C-Arms, Computed Tomography "CT" scanners or Magnetic Resonance Imaging "MRI" scanners beside robotic and navigation techniques (Ahmed et al. [21]). On the other hand, within the traditional surgery, neither surgical robots nor advanced medical imaging equipment are involved, but only the traditional surgical equipment is used. Based on the variety between robotic surgery rooms and traditional surgery rooms, there are some differences between the design of RSW and the design of TSW as detailed later. The details of the comparisons in the scope of the whole ward and internal operating rooms are detailed in the following two sections.

\subsection{The scope of surgery ward: RSW vs. TSW}

Comparative analyses between RSW and TSW have been concluded (as shown in Table 2); this comparative analyses have been conducted in terms of the main architectural characteristics as: a) location, b) the average area of the ward, c) internal zones, d) corridors' type, e) ORs' types, f) the average area of ORs, g) main surgical tools and h) features of the ward. These architectural characteristics can be classified to 2 categories as: a) characteristics mentioned in literature, which were clearly addressed in a relevant previous studies and b) characteristics extracted analytically, which refer to what have been analyzed and extracted by authors based on previous studies.

According to the previous analyses, it can be stated that:

- The relations between RSW and new medical wards (as angiography ward and simulation education center) refers to the need for wider area to include those huge ORs, therefore it needed expensive budget. However, TSW was adjacent to intensive care unit and connected with emergency ward.

- The components of RSW were various that TSW, where the zones of RSW consisted of the 4 main zones: Operating rooms zone, patient zone, stuff zone 
and support service zone, in addition spaces attached to ORs zone. However, TSW consisted of the 4 main zones only.

- The establishment of RSW is required more addition area than TSW by percentage about $35 \%$. For example, the small area for RSW was $900 \mathrm{~m}^{2}$ instead of $600 \mathrm{~m}^{2}$ for TSW

- The zone of spaces attached to ORs includes a set of rooms that are relevant to surgical robots' service as: a) Tele surgery room, b) technical power supply room, c) intraoperative Magnetic Resonance Imaging "MRI"/ Computed Tomography "CT" room d) pump room, e) robot store and f) radiology technicians' office.

- RSW can serve multiple disciplines in one place/ward, so it included on 5 types of ORs: a) digital robotic OR, b) Cardiothoracic hybrid OR, c) Neurosurgery hybrid OR, d) Endovascular hybrid OR and e) Orthopedics hybrid OR, against to 2 types in TSW that are small and large conventional OR.

- Various surgical robots and advanced imaging equipment were used inside the robotic surgery rooms, so, wide work area was required for them and accordingly increasing area of ORs to exceed $70 \mathrm{~m}^{2}$. However, the area of traditional surgery rooms not exceeds $70 \mathrm{~m}^{2}$.

- Digital robots inside ORs require IT infrastructure, so RSW cannot be established at traditional hospitals as a developmental stage.

- Robotic surgery technology helps surgeons to perform extremely complex surgeries, short hospitalization for patient, reduce hospital outgoing, in addition Effectiveness and efficiency in training, teaching and research.

- Spaces' area and cost can be saved in TSW, as well as the safety of intraoperative robot failure. 
Randa M. A. Mahmoud et al., Architectural comparative analyses between robotic surgery wards ......

Table 2.

Architectural comparative analyses between Robotic Surgery Ward and Traditional Surgery Ward. Summary of Ahmed et al. [21]; Akridge [25]; Rostenberg et al. [11, 12]; Gillespie et al. [26]; Schwarz et al. [18]; Harsoor et al.[22]; Health Authority Abu Dhabi guidelines [16]; Beasley [27]; Kpodonu [9,10]; Clausdorff et al. [20]; Facility Guidelines Institute [17].

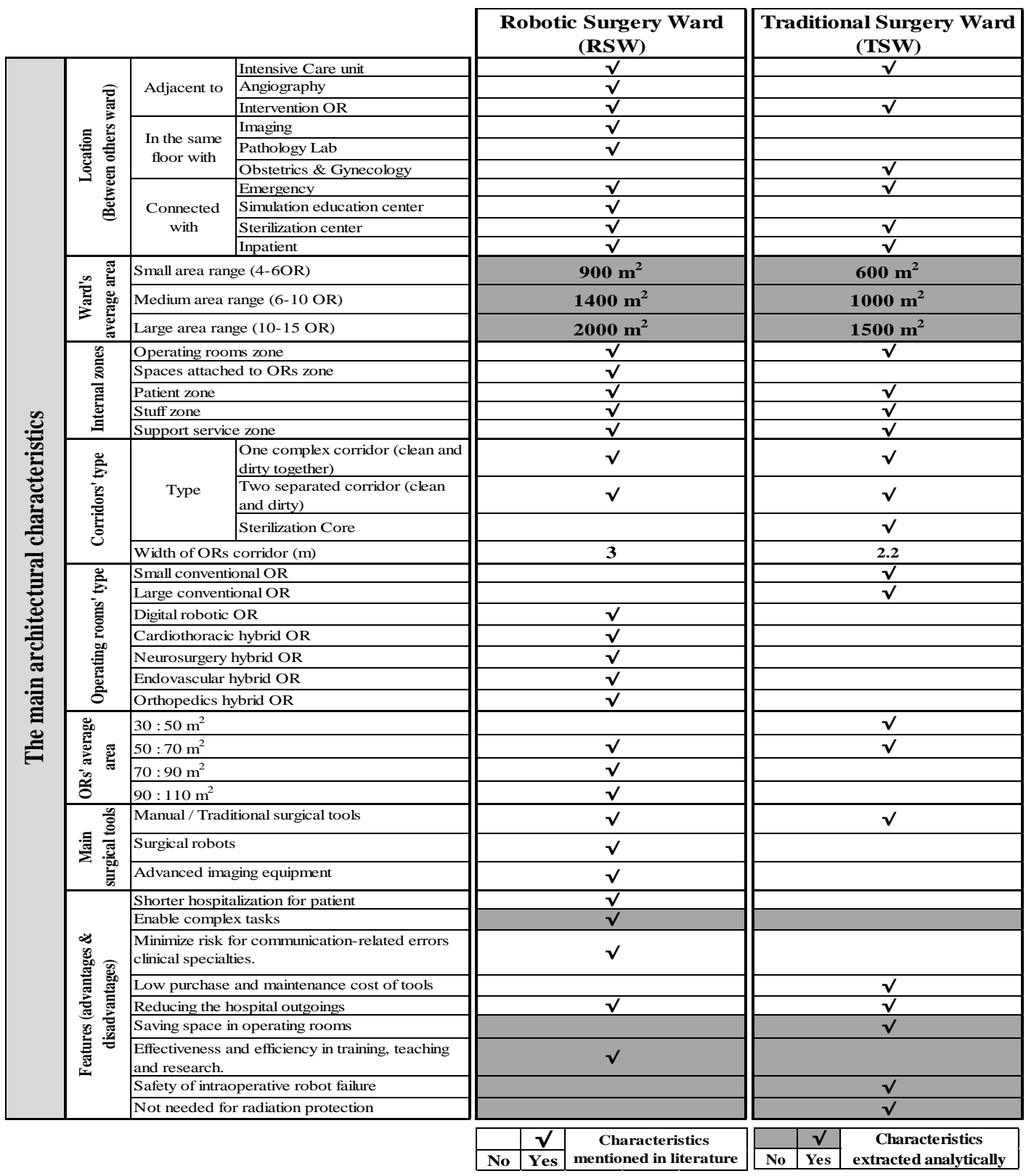

3.2. The scope of operating rooms: robotic surgery rooms vs. traditional surgery rooms

Regarding surgery rooms, Table 3 illustrates detailed comparative analyses between robotic surgery rooms and traditional surgery rooms. The comparative has been conducted based on a set 
of architectural aspects such as: a) main features of robotic surgery rooms and traditional surgery, b) their architectural considerations, c) main architectural details, d) main medical equipment, e) main electronic equipment and f) approximate economic cost. These architectural aspects have been concluded from previous studies as the most common aspects for ORs' design.

Table 3.

Architectural and economic comparative analyses between robotic surgery rooms and traditional surgery rooms. Summary of Rostenberg et al. [11,12]; Kpodonu [9,10]; Emergency Response Centre International Institute [13]; Clausdorff et al. [20]; Facility Guidelines Institute [17]; Health Authority Abu Dhabi guidelines [16]; Ahmed et al. [21]; Harsoor et al. [22]; Siddharth et al. [23]; Mille. [24]; Winkle et al. [28]; Rentz [29]; Dextrom [30]; Sharrock [31]; Richard [32]; Nunez [33]; Block [34]; Wasek [35]

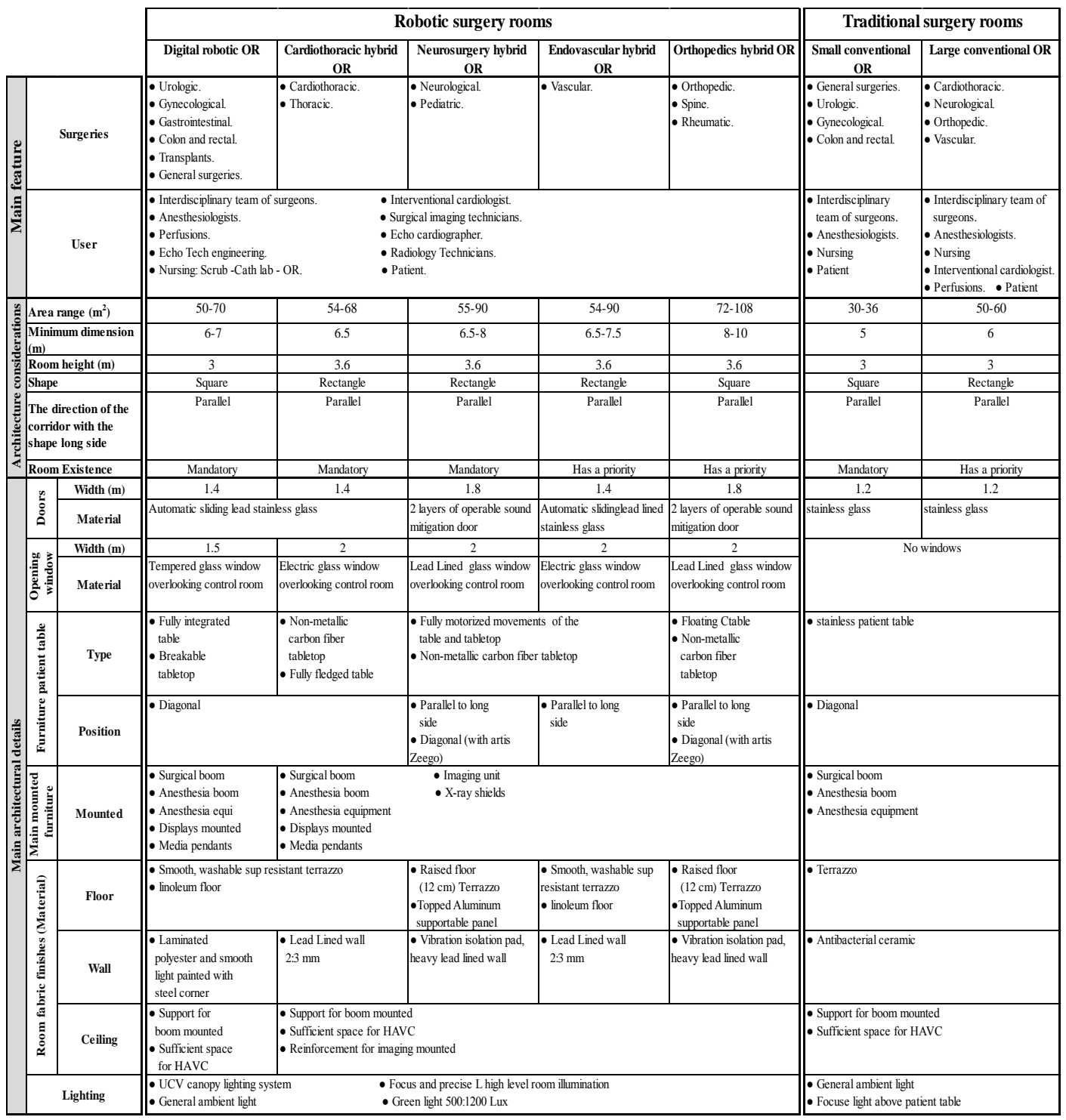


Randa M. A. Mahmoud et al., Architectural comparative analyses between robotic surgery wards ......

\begin{tabular}{|c|c|c|c|c|c|c|c|c|}
\hline & & \multicolumn{5}{|c|}{ Robotic surgery rooms } & \multicolumn{2}{|c|}{ Traditional surgery rooms } \\
\hline & & Digital robotic OR & \begin{tabular}{|c|} 
Cardiothoracic hybrid \\
OR
\end{tabular} & \begin{tabular}{|c|}
$\begin{array}{c}\text { Neurosurgery hybrid } \\
\text { OR }\end{array}$ \\
$\bullet$ Neuromate $=15$ \\
\end{tabular} & \begin{tabular}{|c|}
$\begin{array}{c}\text { Endovascular hybrid } \\
\text { OR }\end{array}$ \\
- PIC robot $=15$
\end{tabular} & Orthopedics hybrid OR & $\begin{array}{c}\text { Small conventional } \\
\text { OR }\end{array}$ & Large conventional OR \\
\hline & $\begin{array}{l}\text { Surgical robots = } \\
\text { Work Area }\left(\mathrm{m}^{2}\right)\end{array}$ & $\begin{array}{ll}\text { - Davinci } & =20 \\
\text { - Zeus robot } & =15 \\
\text { - Rosa robotic } & =15 \\
\text { - Telelap ALF-X } & =40 \\
\text { - TransEnterix } & =40\end{array}$ & - Davinci $=20$ & $\begin{array}{l}- \text { Neuromate }=15 \\
\text { - Rosa roboto }=15\end{array}$ & $\begin{array}{l}\bullet \text { PIC robot }=15 \\
\bullet \text { Davinci }=20\end{array}$ & $\begin{array}{l}\text { - } \mathrm{RIO} \text { robot }=15 \\
\text { - Davinci }=20\end{array}$ & No sur & Irgical robots \\
\hline 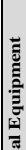 & \begin{tabular}{|l} 
Imagining equipment \\
$=$ \\
Work Area $\left(\mathrm{m}^{2}\right)$ \\
\end{tabular} & Not required & $\begin{array}{l}- \text { Ceiling C-arm }=15 \\
\text { - Floor C-arm }=20 \\
\text { - Artis zee }=15\end{array}$ & $\begin{array}{l}\text { - Artis zee biplane }=20 \\
\text { - Infinx I biplane }=20 \\
\text { - Atris zeego } \quad=30 \\
\text { - Ge-discovery } 730=30 \\
\text { - MRI } \\
25\end{array}$ & $\begin{array}{l}- \text { Ceiling C-arm = } 15 \\
\text { - Floor C-arm = } 20 \\
\text { - Stereotaxis Robotic } \\
\text { Navigation } \\
\text { Technology = }=35\end{array}$ & $\begin{array}{l}- \text { Atris zeego }=30 \\
- \text { Ge-discovery } 730=30 \\
-\mathrm{CT} \\
15\end{array}$ & No imag & ging equipment \\
\hline 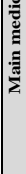 & Medical equipment & $\begin{array}{l}\text { - Anesthesia equipment } \\
\text { - Laparoscopic towers } \\
\text { hold insufflator } \\
\text { - Medical piped gas }\end{array}$ & $\begin{array}{l}\text { - Anesthesia equipment } \\
\text { - Medical piped gas } \\
\text { - Heart-lung by pass } \\
\text { - Construct injector } \\
\text { - Transthoracic \& } \\
\text { intravascular echo }\end{array}$ & $\begin{array}{l}\text { - Anesthesia equipment } \\
\text { - Medical piped gas } \\
\text { - Heart-lung by pass } \\
\text { - Construct injector } \\
\text { - Brain navigation }\end{array}$ & $\begin{array}{l}\text { - Anesthesia equipment } \\
\text { - Medical piped gas } \\
\text { - Heart-lung by pass } \\
\text { - Construct injector } \\
\text { - Transthoracic \& } \\
\text { intravascular echo } \\
\text { - Mobile AS pirate }\end{array}$ & $\begin{array}{l}\text { - Anesthesia equipment } \\
\text { - Medical piped gas } \\
\text { - Construct injector }\end{array}$ & $\begin{array}{l}\text { - Anesthesia equipment } \\
\text { - Medical piped gas } \\
\text { - Construct injector }\end{array}$ & $\begin{array}{l}\text { - Anesthesia equipment } \\
\text { - Medical piped gas } \\
\text { - Construct injector } \\
\text { - Heart-lung by pass } \\
\text { - Brain navigation }\end{array}$ \\
\hline & \begin{tabular}{|l|} 
Traditional \\
equipment
\end{tabular} & $\begin{array}{l}\text { - Built-in glass and stainless } \\
\text { - Equipment carts } \\
\text { - Ceiling mounted boom }\end{array}$ & $s$ cabinets & & & & $\begin{array}{l}\text { - Built-in glass and stain } \\
\text { - Equipment carts } \\
\text { - Ceiling mounted boom }\end{array}$ & nless cabinets \\
\hline & $\begin{array}{l}\text { ICT devices and } \\
\text { system }\end{array}$ & $\begin{array}{l}\text { - Electronic touch panel } \\
\text { - Video camera holder } \\
\text { - Audio-video system }\end{array}$ & $\begin{array}{l}\text { - IT in } \\
\text { - Mon } \\
\text { - Medi }\end{array}$ & $\begin{array}{l}\text { ntegrated equipment } \\
\text { nitors } \\
\text { lia beam }\end{array}$ & & & No IC & CT devices \\
\hline 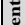 & \begin{tabular}{|l|} 
Media equipment \\
\end{tabular} & - Media beam (power -netv & work - Video) & ouch screen monitors mounte & ed and on wall & & No med & dia equipment \\
\hline 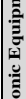 & $\begin{array}{l}\text { Picture Archiving } \\
\text { and Communication } \\
\text { System (PACS) }\end{array}$ & \begin{tabular}{|l} 
- Ultrasound (IVAS) on wa \\
- OR integrated system \\
- RLTS \& RFID system \\
- Data management
\end{tabular} & & & & & NoPA & ACS system \\
\hline 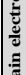 & Electrical power & \begin{tabular}{|l} 
- 24 electric outlets at heigh \\
- Emergency generator wit
\end{tabular} & $\begin{array}{l}\text { ht } 1.5 \mathrm{~m} \\
\text { h automatic } 2 \text { ways change o }\end{array}$ & over facility & & & $\begin{array}{l}\text { - } 12 \text { electric outlets at he } \\
\text { - Emergency generator } \\
\text { change over facility }\end{array}$ & with automatic 2 ways \\
\hline $\bar{\Sigma}$ & General Equipment & $\begin{array}{l}\text { - Electrical installing and he } \\
\text { - Medical gases piped }\end{array}$ & $\begin{array}{ll}\text { eating channels } & \bullet \text { Fire } \\
& \bullet \text { Air ch }\end{array}$ & $\begin{array}{l}\text { extinguishers } \\
\text { hanger and optimal temperat }\end{array}$ & & & $\begin{array}{l}\text { - Fire extinguishers } \\
\text { - Electrical installing and } \\
\text { - Air changer and optim }\end{array}$ & $\begin{array}{l}\text { - Medical gases piped } \\
\text { d heating channels } \\
\text { nal temperature }\end{array}$ \\
\hline (A) & \begin{tabular}{|l|}
$\begin{array}{l}\text { Surgical robots cost } \\
(\mathrm{M} \$)\end{array}$ \\
\end{tabular} & $1.5-3$ & $1.5 \cdot 2.25$ & $0.6-0.8$ & $1.2 \cdot 2.25$ & $1.0-2.25$ & No sur & rgical robots \\
\hline 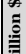 & $\begin{array}{l}\text { Imaging equipment } \\
\operatorname{cost}(M \$)\end{array}$ & Not required & $0.3-1.2$ & $1.2-2.3$ & $1.5-2.85$ & $1.5 \cdot 2.25$ & No imagi & ging equipment \\
\hline$\underline{t}$ & $\begin{array}{l}\text { Other equipment } \\
\text { and furniture (M \$) }\end{array}$ & $1.0-1.6$ & $1.0-1.3$ & $1.0-1.3$ & $1.0-1.3$ & $1.0-1.6$ & $0.35-0.5$ & $0.5-0.8$ \\
\hline 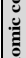 & \begin{tabular}{|l|} 
Cost of space \\
$\left(\$ / \mathrm{m}^{2}\right)$
\end{tabular} & $500 \$ / \mathrm{m}^{2}$ & $1000 \$ / \mathrm{m}^{2}$ & $1000 \$ / \mathrm{m}^{2}$ & $1000 \$ / \mathrm{m}^{2}$ & $1000 \$ \mathrm{~m}^{2}$ & $500 \$ / \mathrm{m}^{2}$ & $500 \$ / \mathrm{m}^{2}$ \\
\hline : & \begin{tabular}{|l} 
Ceiling \\
reinforcement (M \$)
\end{tabular} & No needed for it because & 0.08 & 0.08 & 0.08 & 0.08 & $\begin{array}{r}\text { No needed for it becal } \\
\text { re }\end{array}$ & $\begin{array}{l}\text { ause imaging mounted is not } \\
\text { equired }\end{array}$ \\
\hline , & $\begin{array}{l}\text { Lead lined shielding } \\
\text { wall (M \$) }\end{array}$ & $\begin{array}{l}\text { required } \\
\text { red }\end{array}$ & 0.1 & 0.1 & 0.1 & 0.1 & $\begin{array}{r}\text { No needed for it becal } \\
\text { re }\end{array}$ & $\begin{array}{l}\text { ause imaging mounted is not } \\
\text { equired }\end{array}$ \\
\hline & $\begin{array}{l}\text { Total approximate } \\
\text { cost M \$ }\end{array}$ & 2.7 - 3.6 M\$ & $3.1-3.9 \mathrm{M} \$$ & $3.1-3.8 \mathrm{M} \$$ & $3.0-4.9 \mathrm{M} \$$ & $4.4-5.8 \mathrm{M} \$$ & $0.5-0.8 \mathrm{M} \$$ & $0.7-1.0 \mathrm{M} \$$ \\
\hline & $\begin{array}{l}\text { Te: } \\
\text { T: Information Technolog } \\
\text { MRI : Magnetic Resonanc } \\
\text { Total cost of OR is include } \\
\text { The economic cost was est }\end{array}$ & $\begin{array}{l}\text { - Imaging } \quad \bullet \mathrm{CT} \text { : Inform } \\
\text { d surgical robot cost }+ \text { imagin } \\
\text { timated based on internationa }\end{array}$ & $\begin{array}{l}\text { ation and Communications T } \\
\text { ed Tomography } \\
\text { lg equipment cost + other me } \\
1 \text { prices of robots, equipment }\end{array}$ & $\begin{array}{l}\text { - PACS : Pic } \\
\bullet \text { PET : Posi } \\
\text { dical equipment cost + (cost } \\
\text { and construction. }\end{array}$ & $\begin{array}{l}\text { cture Archiving and Commur } \\
\text { itron Emission Tomography } \\
\text { of space } X \text { area of } \mathrm{OR})+\mathrm{c}\end{array}$ & $\begin{array}{l}\text { nication System } \bullet \text { HVAC } \\
\text { eiling reinforcement cost + le }\end{array}$ & $\begin{array}{l}: \text { Haeting, Ventilation and } \\
\text { ad line shielding cost }\end{array}$ & d Air Conditioning \\
\hline
\end{tabular}

Based on the previous analyses, it can be stated that:

- The number of users inside robotic surgery rooms was twice in traditional surgery rooms, so wide work area was required.

- Robotic surgery rooms had wide area range between $50: 110 \mathrm{~m}^{2}$ because of the required work area of robots that varied between 15:40 $\mathrm{m}^{2}$. However the area of traditional surgery rooms did not exceeded $60 \mathrm{~m}^{2}$.

- The height of some robotic surgery rooms was higher than traditional surgery rooms due to the mounted of imaging equipment and other media mounted. 
- The patient table inside robotic surgery rooms should be movable and suitable with robots and imaging equipment movement. But, stainless patient table was used inside traditional surgery rooms

- Because of the usage of surgical robots and imaging equipment inside ORs, radiation protection material must be used as: heavy lead lined wall and vibration isolation pad.

- Also, the ceiling should be reinforcement for imaging mounted and the floor should be raised floor with aluminum panels to allow the electronic and electrical supplies. However, terrazzo floor was used for traditional surgery rooms.

- Automatic sliding lead stainless doors were used inside robotic surgery rooms to protect users from the Radiation of imaging equipment.

- There was not any window in traditional surgery rooms, although lead lined glass window are been in robotic surgery ward and overlooking control room

- According to the medical equipment, the surgeons inside traditional surgery rooms use medical and traditional equipment manually. Conversely, surgeons inside robotic surgery rooms use tele-manipulator or robot control tool.

- The main electronic equipment inside traditional surgery rooms limited to electrical power supplies and general equipment as medical gases. But complex electronic equipment were required inside robotic surgery rooms as: Information and Communications Technologies devices and system, media equipment and Picture Archive and Communication System.

- Based on the economic comparative analyses, it is found that establishment of Orthopedics hybrid OR was the most expensive because of the wide required area and the high price of the used surgical robots and imaging equipment. The cost of digital robotic OR establishment was cheap in order to no use of imaging equipment.

- Small and large conventional ORs' cost was the cheapest due to no required for surgical robots or imaging equipment

\section{Proposed possible designs for robotic surgery ward and traditional surgery ward: architectural applications}

By following the previous main architectural aspects, many design alternatives of RSWs and TSWs could be proposed to help designers to work on their RSW and TSW designs. Accordingly, a set of 12 design alternatives of RSWs and TSWs have been presented as an application as shown in Table 4. All design alternatives have been proposed as rectangular shapes to simplify design process without any relations with other external wards. Also, previous architectural and economic considerations (came from the comparative analyses) have been used in the conducting the proposed designs. Then, the alternatives have been distributed among 3 groups are: a) the small area alternatives (4-6 ORs), b) the medium area alternatives (6-10 ORs) and c) the large area alternatives (10-15 ORs). Finally, the properties of design alternatives have been compared.

With analyzing and comparing the proposed alternatives of RSWs and TSWs, it obvious that RSWs had wide area that reached to $900 \mathrm{~m}^{2}$ with just 4-6 ORS as obvious in alternatives (1) and (2), while TSWs are smaller and cheaper as in alternatives (3) and (4). In alternative (7), the support service zone was integrated with ORs to establish sterilization core, but it cannot be implemented in RSWs because there are control room for each OR. Alternative (10) included 14 wide ORs that required wide area of ward overall, hence waste area was created in other zones. Although alternative (12) contained the same ORs number, the 
Randa M. A. Mahmoud et al., Architectural comparative analyses between robotic surgery wards .......

TSW's area was saved. As shown in Table 4, the comparative between the different alternatives can help designers to determine the appropriate required area for RSW or TSW based on ORs numbers. Also, the average total cost of ORs has been estimated as initial budget, whereas the cost of ORs can be considered the most important ratio of the whole budget of the surgery ward. Mostly, RSW designs were different than TSW in terms of required area, zones and cost. Hence, the design alternatives could be considered as initial designs that can help designers within the design process.

\section{Table 4.}

A set of 12 possible design alternatives of Robotic Surgery Ward and traditional Surgery Ward as applications

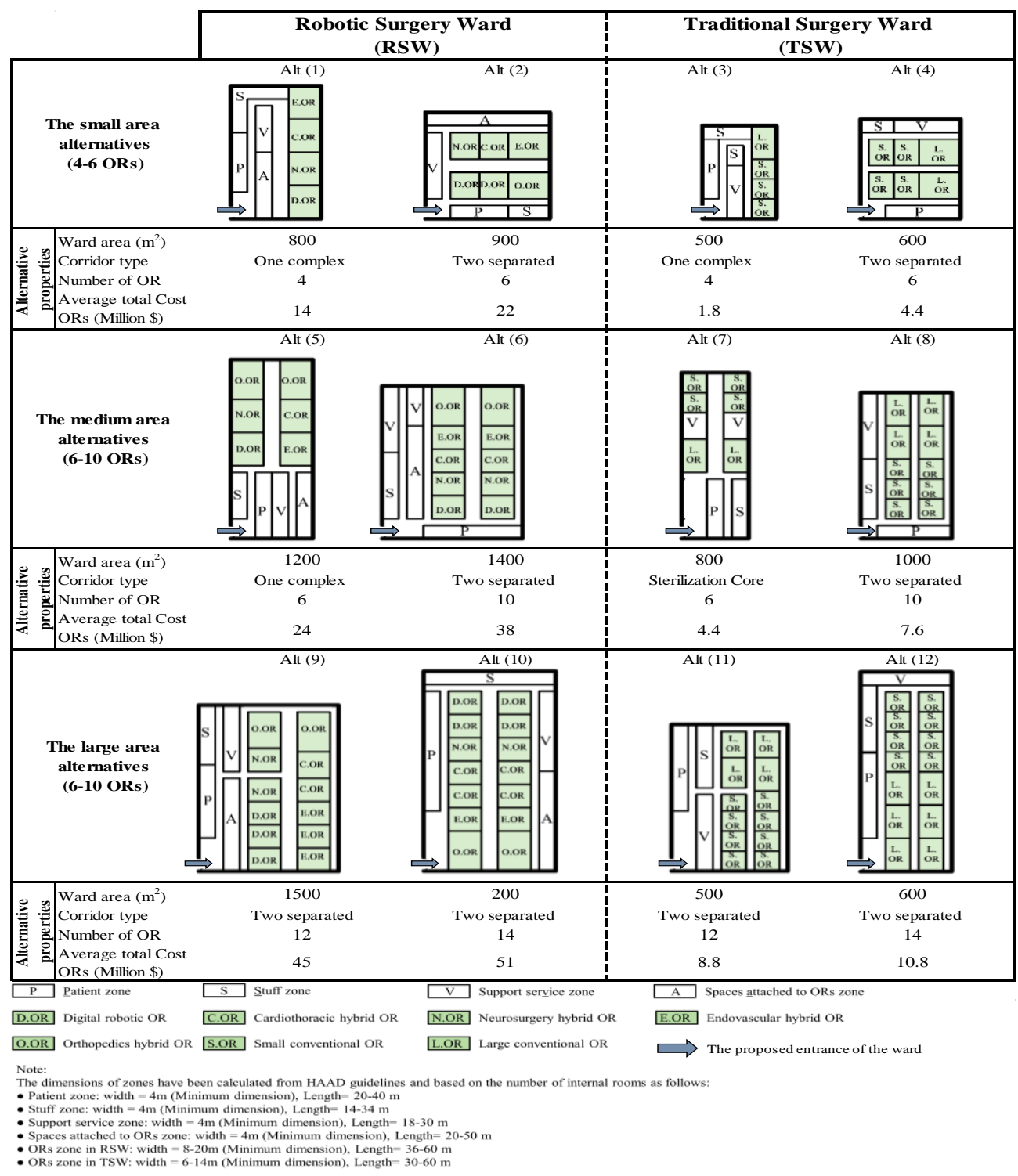




\section{Conclusion}

This paper presented comparative analyses between Robotic Surgery ward (RSW) and Traditional Surgery Ward (TSW). It started with focusing on the effect of healthcare advanced technologies on the design of digital hospital and illustrated the architectural considerations of digital hospital's rooms which have these healthcare advanced technologies. It was found that robotic surgery ward is one of the best demonstration of integrating healthcare advanced technologies. Subsequently, the comparative analyses between RSW and TSW has been concluded in terms of the main architectural characteristics as: a) location, b) the average area of the ward, c) internal zones, d) corridors' type, e) ORs' types, f) the average area of ORs, g) main surgical tools and h) features of the ward. These architectural characteristics can be classified to 2 categories as: a) characteristics mentioned in literature and b) characteristics extracted analytically. Finally, different design alternatives of RSWs and TSWs have been presented and compared as an application. Consequently, the appropriate required area and initial budget of RSW or TSW could be determined.

It can observe that the establishment of RSW needs wide area to include those huge ORs, accordingly it needs expensive budget. The components of RSW were various than TSW, where the zones of RSW consisted of the 4 main zones: Operating rooms zone, patient zone, stuff zone and support service zone, in addition spaces attached to ORs zone. Also, RSW can serve multiple disciplines in one place/ward, so it included 5 types of ORs against to 2 types in TSWs. A wide work area in RSWs was required because of the usage of surgical robots and imaging equipment. Mostly, Robotic surgery technology helps surgeons to perform extremely complex surgeries and reduce hospital outgoing, but spaces' area and cost can be saved in TSWs. As well, detailed comparative analyses between robotic surgery rooms and traditional surgery rooms has been conducted based on a set of main architectural aspects as (features of robotic surgery rooms and traditional surgery, their detailed architectural considerations, , medical and electronic equipment and approximate economic cost. As a result, robotic surgery rooms had wide area range between $50: 110 \mathrm{~m}^{2}$ because of the required work area of robots that varied between 15:40 $\mathrm{m}^{2}$, as well as their specific height was around $3.6 \mathrm{~m}$. Because of the usage of surgical robots and imaging equipment inside ORs, radiation protection material must be used. Also, complex electronic equipment were required inside robotic surgery rooms as: Information and Communications Technologies devices and system, media equipment and Picture Archive and Communication System. Based on the economic comparison, the establishment of Orthopedics hybrid OR was the most expensive OR because of the wide required area, while the digital robotic OR establishment was the cheapest, but the cost of traditional surgery rooms' establishment was $400 \$ / \mathrm{m}^{2}$ in addition surgical equipment cost so the total cost did not exceed 1.0 million Dollar.

Hence, the proposed designed alternatives could be considered as initial designs that can help designers within the design process for their both RSW and TSW cases. The study can be extended to include the details of all RSW rooms and calculation of the total cost of the ward. Also, a design framework for RSW can be proposed to facility the design process and save designers' time and effort. In addition to a computational implementation can be developed based on the design framework to generate design alternatives of RSW computationally. 
Randa M. A. Mahmoud et al., Architectural comparative analyses between robotic surgery wards ......

\section{REFERENCES}

[1] Kilic, T. (2016).Digital hospital an Example of best practice. International Journal of Health Science Research and Policy. Volume 1 Issue 22016

[2] Reffat, R.M. (2014). Hospitals of the Future using Advanced Technologies. Second Umran Saudi forum for planning and design of hospitals. Organized by the Health Buildings Architects Division, affiliated to the Saudi Society, the forum is taking place at the RitzCarlton Hotel, Riyadh in April 2014.

[3] Korea Digital Hospital Export Agency (KOHEA). (2015).Concept and Advantage of Digital Hospital. Available via: http://eng.kohea.co.kr/hospital_concept/hospital_mobile. Accessed at 17 February 2019.

[4] Courbis, T. (2016).Digital hospital: Evidence based design. Finances Hospitalières. April 2016.

[5] Sprow, R. (2012) Planning Hospitals of the Future, In G. D. Kunders (ed.), Designing Hospitals of the Future, Prism Publications.

[6] Martin, C. (2011). Telemedicine Room Design; Program Guide. A Publication of: California Telemedicine and eHealth Center

[7] Kenyon, A. (2015). Medical Simulation: Designing for the Future. Progress in Journal of Study to solutions vol (4). Accessed at 2 May 2018

[8] Matthew J.Z, Matthew T.G, John J.K (2011) Robotic Instrumentation, Personnel and Operating Room Setup. Atlas of Robotic Urologic Surgery DOI 10.1007/978-1-60761-0267_2. J Springer Science: 15-30.

[9] Kpodonu, J. (2012). The Cardiovascular Hybrid Surgical Room: Evolving into the Future of Cardiovascular Surgery. Article Published on March 2012. Article publisher is cath lab digest web site. Available via: http://www.cathlabdigest.com/articles/Cardiovascular-HybridSurgical-Room-Evolving-Future-Cardiovascular-Surgery. Accessed at 1 May 2018.

[10] Kpodonu, J. (2015).Cardiovascular Hybrid OR: The Travelled Road. Article Published on Jun 11, 2015. Available via: https://www.slideshare.net/JacquesKpodonu/advancedcardiovascular-surgery-hybrid-operating-room-nuts-bolts. Accessed at 17 May 2018. Accessed at 2 January 2019.

[11] Rostenberg, B, Barach, P.R. (2012).Design of cardiovascular operating rooms for tomorrow's technology and clinical practice - Part two. Progress in Pediatric Cardiology; 33: 57-65.

[12] Rostenberg, B, Barach, P.R. (2011).Design of cardiovascular operating rooms for tomorrow's technology and clinical practice - Part one. Progress in Pediatric Cardiology; 32: 121-128.

[13] Emergency Response Centre International (ECRI) Institute. (2013). Hybrid Operating Rooms: with a focus on Endovascular Hybrid ORs Planning guidelines, pricing, and procurement trends. By ECRI Institute's SELECT plus ${ }^{\mathrm{TM}}$ Market Analytics. Available via: https://www.aiic.it/wp-content/uploads/2016/01/ECR13-

MS13084_HybridOR_Market_Analytics_Snapshot.pdf . Accessed at 4 May 2018.

[14] Gow, C, Byrd, B. (2013). Redefining The Operating Room.Article Published on 20 August, 2013. Article publisher is health care design magazine web site. Available via: http://www.healthcaredesignmagazine.com/article/redefining-operating-room. Accessed at 16 March 2018.

[15] Michael J.E, Edward R.S, Darren B.O (2014) the Hybrid Neurovascular Operating Room. J. Springer Science+Business: 301-310.

[16] HAAD Guidelines for Health Facility Design, Approvals Construction and Consultant Prequalification, Part B - Health Facility Briefing \& Design, Volume 1 Including Functional Planning Units, Version 3.3, edition April 2014. https://www.haad.ae/haad/tabid/1125/Default.aspx. Accessed at 25 March 2018

[17] The Facilities Guidelines Institute (FGI), Guidelines for Design and Construction of Hospitals and Outpatient Facilities, USA (2014). Available via: https://ams.aha.org/eweb/DynamicPage.aspx?WebCode=Prod DetailAdd\&ivd_prc_prd_key=8d03858d980b-4a66-9d68-71dabd5fca14 . Accessed at 25 March 2018. 
[18] Schwarz, K, Bastien, K. (2014). Toward a regional healthcare architecture for Saudi Arabia and the Middle East. Second Saudi forum for planning and design of hospitals. Organized by the Health Buildings Architects Division, affiliated to the Saudi Umran Society, the forum is taking place at the Ritz-Carlton Hotel, Riyadh in April 2014

[19] Weremeychik, E. (2014).How To Design a 'Smart' Hospital. Article Published on 17 Dec 2014. Article publisher is healthcare design magazine. Available via: https://www.healthcaredesignmagazine. com/trends/construction-engineering/how-design-smart-hospital/ . Accessed at 10 March 2018

[20] Clausdorff, L.F, Bulittab, C. (2013). Hybrid Theaters: Technical Design and Technology, from Planning to Reality. Pan Vascular Medicine: Springer-Verlag Berlin Heidelberg 2013

[21] Ahmed, S, Khanam, N.H. (2017). Hybrid Operating Room. Article Published on Jan 24, 2017. Available via: https://www.slideshare.net/hamzaaaaaah/hybrid-operating-room . Accessed at 16 March 2018.

[22] Harsoor, S.S, Bhaskar, S.B. (2007). Designing an ideal operating room complex. Indian Journal of Anesthesia; 51 (3): 193-199

[23] Siddharth, V, Kant, S, Chandrashekhar, R, and Gupta, S.K. (2014). Planning premises and design considerations for hybrid operating room. International Journal of Research Foundation of hospital \& Healthcare Administration; 2(1): 50-56

[24] Mille, T. (2012). The Hybrid challenge. Article Published on Jan 7, 2012. Article publisher is Health facilities web site.

[25] Akridge, J. (2013). Advanced operations fuel surgical suite innovations. Article Published on Jan, 2013. Article publisher is health care purchasing news web site. Available via: https://www.hpnonline.com/inside/2013-01/1301-OR-Suites.html. Accessed at 3 March 2019.

[26] Gillespie, D, Otte, J. (2017). The Remote Control Hospital: Healthcare in the Age of Robotic Medicine. Healthcare design conference-Orlando. November 2017

[27] Beasley, R.A. (2012). Medical Robots: Current Systems and Research Directions. Progress in Journal of Robotics Volume 2012, 1:14

[28] Winkle, R.A, Mead, R.H, Engel, G, Kong, M.H, Patrawala, R.A. (2013). Physiciancontrolled costs: the choice of equipment used for atrial fibrillation ablation. Progress in Journal of Interventional Cardiac Electrophysiology 36(2), 157-165.

[29] Rentz, S. (2018). MRI Machine Cost and Price Guide. Article Published on Apr 3, 2018. Article publisher is block imaging web site. Available via: https://info.blockimaging.com/bid/92623/mrimachine-cost-and-price-guide. Accessed at 15 Nov 2018.

[30] Dextrom, K. (2018). Digital Cath Lab Equipment Cost Price Guide. Article Published on Apr 23, 2018. Article publisher is block imaging web site. Available via: https://info.blockimaging.com/bid/96958/digitalcath-lab-equipment-cost-price-guide. Accessed at 15 Nov 2018.

[31] Sharrock, C. (2018). C-arm Cost Price Guide. Article Published on Mar 7, 2018. Article publisher is block imaging web site. Available via: https://info.blockimaging.com/c-armcost-price-guide. Accessed at 15 Nov 2018.

[32] Richard, T. (2014). How much does a Ge Innova cath lab cost. Article Published on May 19, 2014. Article publisher is block imaging web site. Available via: https://info.blockimaging.com/howmuch-does-a-ge-innova-cath-lab-cost. Accessed at 15 Nov 2018.

[33] Nunez, J. (2018). How much does a PRT CT scanner cost. Article Published on Apr 6, 2018. Article publisher is block imaging web site. Available via: https://info.blockimaging.com/bid/68875/howmuch-does-a-pet-ct-scanner-cost . Accessed at 15 Nov 2018

[34] Block, J. (2015). GE Discovery IGS 730 vs. GE Innova IGS 530. Article Published on Oct 2, 2015. Article publisher is block imaging web site. Available via: https://info.blockimaging.com/gediscovery-igs-730-vs.-ge-innova-igs-530 . Accessed at 15 Nov 2018.

[35] Wasek, S. (2018). Cost to build: Sample 2-OR ASC. Article Published on March 1, 2018. Article publisher is beckersasc web site. Available via: https://cdn2.hubspot.net/hub/54848/file14572151-pdf/docs/2008_0910_cost_to_build_sample_2-or_asc_beckers_asc_review.pdf.

Accessed at 1 June 2019. 


\section{تحليل معماري مقارن بين تصميم جناح الجراحات الروبوتية بالمستشفيات ألرقمية وجناح الجراحات بالمستثفيات التقليدية التربية}

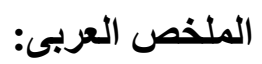

بدأ مؤخر أ تعزيز التكامل بين التقنيات الجديدة لخدمات الرعاية الصحية، وذلك لكونها ضروبات الرية الرية وفعالة

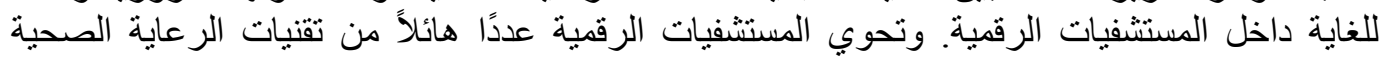

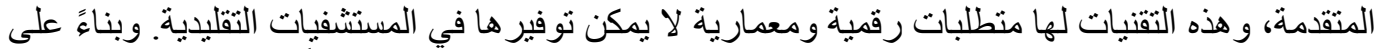

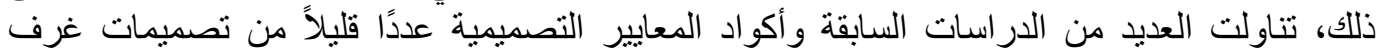

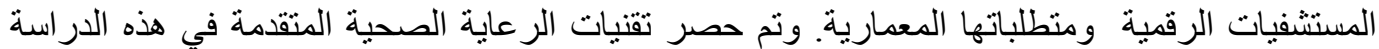

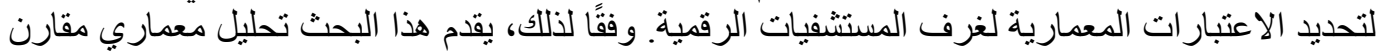

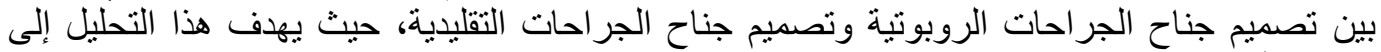

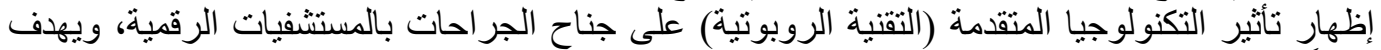

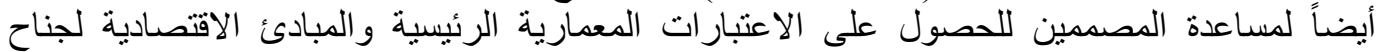

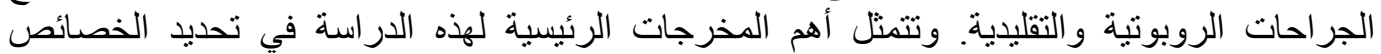

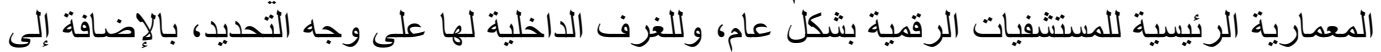

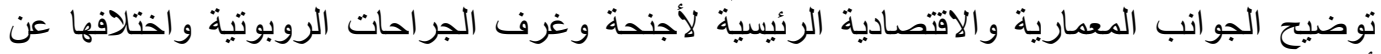

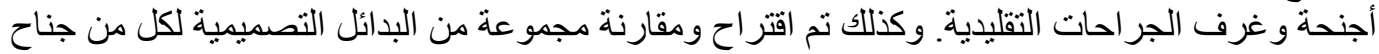

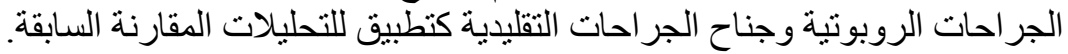

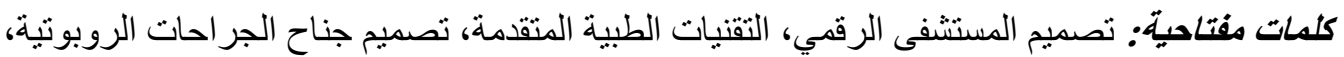
الاعتبار ات المعمارية والإقتصادية. 